\section{Two cases of possible neuro-Sweet disease with meningoencephalitis as the initial manifestation}

\author{
Go Makimoto, ${ }^{1}$ Yasuhiro Manabe, ${ }^{1}$ \\ Chizuru Yamakawa,1 Daiki Fujii,1 \\ Yasuko Ikeda-Sakai,1 Hisashi Narai,1 \\ Nobuhiko Omori,1 Koji Abe² \\ 1Department of Neurology, National \\ Hospital Organization Okayama Medical \\ Center; 2Department of Neurology, \\ Graduate School of Medicine, Dentistry \\ and Pharmaceutical Sciences, Okayama \\ University, Okayama, Japan
}

\begin{abstract}
We report 2 cases that were considered to be neuro-Sweet disease. They initially manifested with meningoencephalitis and no skin lesions, and rapidly improved with corticosteroid therapy. In both cases, patients complained of meningitic symptoms such as fever and headache, and HLA-B54 and -Cwl turned out to be positive over the clinical course. Cerebrospinal fluid analysis showed increased levels of lymphocytes and protein. In case \#1, fluid-attenuated inversion recovery (FLAIR), magnetic resonance imaging (MRI) and diffusion-weighted images (DWI) showed high-intensity signals in the right dorsal medulla oblongata, bilateral dorsal midbrain, and left thalamus. In case \#2, FLAIR and DWI showed high-intensity signals in the bilateral cerebellar cortex and left caudate nucleus. Symptoms and MRI images were markedly improved in both cases after corticosteroid pulse therapy. According to published diagnostic criteria, these 2 cases were considered possible neuro-Sweet disease. These cases suggest that the combination of meningoencephalitis and HLA specificity is important to consider the possibility of neuro-Sweet disease, even without skin lesions.
\end{abstract}

\section{Introduction}

Sweet disease is a systemic disease characterized by fever, peripheral blood leukocytosis, and tender, elevated, erythematous plaques on the face, extremities, and elsewhere.1,2 Sweet disease with central nervous system involvement has been reported as neuro-Sweet disease (NSD). ${ }^{3}$ Cases of NSD presenting with meningoencephalitis have been reported, but skin symptoms usually precede or coincide with neurological symptoms in most cases, and the neurological symptoms rarely precede the cutaneous symptoms. We report 2 patients who initially presented with meningoencephalitis and in whom NSD was suspected from the results of HLA typing, although no skin symptoms were observed.

\section{Case \#1}

The patient was a 51-year old woman whose chief complaints were an unsteady gait, headache, and fever. Her family history was unremarkable. She had undergone surgery for a benign right femoral tumor at the age of 42 , and had been suspected of having a brainstem glioma (which subsided after steroid therapy) at the age of 44 . Eight days before admission to our department, she started to develop a headache on awakening, followed by dizziness, an unsteady gait, and double vision. The next day, she had a fever of $39-40^{\circ} \mathrm{C}$, and visited a local clinic. Since brain MRI showed a brainstem lesion, she was admitted to our hospital. On admission, her temperature was $37.2^{\circ} \mathrm{C}$, and blood pressure 122/76 mmHg. No remarkable features were observed in heart, lungs, and abdomen. There were no skin lesions. Needle reaction was negative. Neurological examination showed a clear consciousness, horizontal nystagmus on lateral gaze to the right, an unpleasant sensation on the right side of the face, and dysarthria. Barré's sign was positive in the right upper limb, and Mingazzini's sign was positive in the right lower limb. Tendon reflexes were somewhat increased in the right arm and leg, but no pathological reflexes were noted. The finger-tonose test was poorly performed on the right side. She had a cerebellar ataxic gait, and could not perform tandem gait. No abnormalities were seen in the autonomic nervous system. There was no nuchal rigidity or Kernig's sign.

Laboratory findings on admission were: erythrocyte sedimentation rate $25 \mathrm{~mm} / \mathrm{h}$, increased white blood cell count $9,000 / \mathrm{mL}$ (77.9\% neutrophils), and hepatic dysfunction with elevated AST (123 IU/L) and ALT (397 IU/L). Serum antibody titers against various viruses indicated past exposure to them. Antinuclear antibody was negative. ACE and lysozyme levels were within normal limits. The s-IL-2R level was $272 \mathrm{U} / \mathrm{mL}$ (standard value 145-519 U/mL). The Mantoux test was negative. Cerebrospinal fluid examination showed a normal initial pressure, cell count of 129/mL (80 monocytes and 49 polymorphs), protein of $72 \mathrm{mg} / \mathrm{dL}$, and glucose of $53 \mathrm{mg} / \mathrm{dL}$ (fasting blood glucose $108 \mathrm{mg} / \mathrm{dL}$ ). The IL-6 level was $5.1 \mathrm{pg} / \mathrm{mL}$, the myelin basic protein level was normal, and the oligoclonal IgG band was negative. FLAIR and diffusion-weighted brain MRI showed high signal intensity areas in the right
Correspondence: Yasuhiro Manabe, Department of Neurology, National Hospital Organization Okayama Medical Center, 1711-1 Tamasu Kitaku, Okayama, Okayama 701-1192, Japan.

Tel. +86.294.9911 - Fax: +86.294.9255.

E-mail: ymanabe@okayama3.hosp.go.jp

Key words: neuro-Sweet disease, meningoencephalitis, neuro-neutrophilic disease.

Received for publication: 4 August 2011.

Revision received: 20 October 2011.

Accepted for publication: 17 January 2012.

This work is licensed under a Creative Commons Attribution NonCommercial 3.0 License (CC BYNC 3.0).

(C) Copyright G. Makimoto et al., 2012

Licensee PAGEPress srl, Italy

Neurology International 2012; 4:e5

doi:10.4081/ni.2012.e5

dorsal medulla oblongata, ventral pons (predominantly on the left side), bilateral midbrain, and left hypothalamus, with no contrast enhancement (Figure 1A and B). ADC values were increased in these areas (Figure 1C).

After admission, multiple demyelinating lesions were observed mainly in the brainstem, strongly suggesting a non-infectious autoimmune disease; therefore, steroid pulse therapy with $1 \mathrm{~g} /$ day of methylprednisolone (mPLS) for three days was started on the day of admission. Thereafter, the neurological symptoms gradually improved, and the cerebrospinal fluid cell count decreased. MRI showed shrinkage of the high-density areas. On Day 12 after admission, she was found to be negative for HLA-B51 and positive for HLAB54 and -Cw1. After 3 courses of steroid pulse therapy, she was switched to $30 \mathrm{mg} /$ day of oral PSL on the Day 19 after admission, which was then tapered. A brain MRI on Day 20 after admission showed the disappearance of the lesions (Figure 1D). The patient experienced no disease recurrence, and was discharged on Day 21 after admission. Subsequently, she was followed-up as an outpatient, and steroid therapy was discontinued three months later. At present, two years after onset, she shows no recurrence of symptoms or skin lesions.

\section{Case \#2}

The patient was a 42-year old woman whose complaint was of headache and fever. The family history showed that her grandfather had died of pancreatic cancer. She had a history of surgery for acute appendicitis at the age of 16 . She presented with common cold-like symptoms, such as headache and fever, two weeks 
before admission to our department, and had visited a local clinic, where she had been prescribed antibiotics (details are unknown). Since the symptoms did not improve, she was admitted to our hospital. On admission, her temperature was $39.3^{\circ} \mathrm{C}$, and blood pressure $120 / 72 \mathrm{mmHg}$. There were no remarkable features in heart, lungs, or abdomen. There were no skin lesions. The needle reaction was negative. Neurological examination revealed the disturbance of consciousness (JCSI-2). There were no cranial nerve, motor, or sensory abnormalities. The tendon reflexes were normal, and no pathological reflexes were noted. She had a cerebellar ataxic gait, and could not perform tandem gait. The autonomic nervous system showed no abnormalities. Nuchal rigidity was noted, but Kernig's sign was negative.

Laboratory examination on admission revealed a white blood cell count of $5300 / \mathrm{mL}$, and no biochemical abnormalities. Serum antibody titers against various viruses indicated past exposure to them. Anti-nuclear antibody was negative. Cerebrospinal fluid examination showed a normal initial pressure, cell count of 332/mL (328 monocytes and 4 polymorphs), protein of $371 \mathrm{mg} / \mathrm{dL}$, and glucose of $28 \mathrm{mg} / \mathrm{dL}$ (fasting blood glucose, $117 \mathrm{mg} / \mathrm{dL}$ ). Brain MRI revealed no abnormal signal.

After admission, she was initially suspected of having bacterial or viral meningitis, and was started on an intravenous drip infusion of ceftriaxone ( $4 \mathrm{~g} /$ day) and acyclovir (750 mg/day). However, the fever did not improve, and the level of consciousness deteriorated to JCS 10 or higher. On Day 12 after admission she was found to be negative for HLA-B51 and positive for HLA-B54 and -Cw1, which suggested neuroSweet disease. The next day, she was started on steroid pulse therapy ( $1 \mathrm{~g} /$ day of mPLS for 3 days). On Day 13 after admission, FLAIR and diffusion-weighted brain MRI showed highsignal-intensity areas in the bilateral cerebellar hemispheres and left caudate nucleus (Figure 2A,B). She received a total of three courses of steroid pulse therapy. Since her consciousness level and neurological symptoms, including right hemiparesis and ataxic gait, gradually improved, she was discharged on Day 27 after admission. FLAIR brain MRI findings observed on Day 13 after admission had disappeared on Day 27 after admission (Figure 2C). Subsequently, she was followed-up as an outpatient. At present, one year after onset, only slight paralysis of the right arm and leg remains, and she has experienced no recurrence of symptoms or skin lesions.

\section{Discussion}

The two patients initially presented with meningitis, but had no skin lesions. They responded well to steroid therapy, resulting in a rapid improvement of the clinical symptoms and imaging findings. During their clinical course, they were found to be positive for HLAB54 and -Cw1. Since Patient \#1 had a history of steroid therapy for similar symptoms eight years previously, a demyelinating disease was suspected. However, this was excluded because the clinical and laboratory findings were atypical in that the cerebrospinal fluid cell count increased, the lesions were confined to the brainstem, no contrast enhancement was observed, and a high fever of $39-40^{\circ} \mathrm{C}$ was noted. Although she had no skin lesions, she responded to steroids. Therefore, in view of the diagnostic criteria for NSD proposed by Hisanaga et al. ${ }^{3}$ in 2005 , she was considered to have possible NSD. Patient \#2 presented with clinical features of meningitis on admission, but she was resistant to antibiotics and antiviral drugs. This patient was also considered to have possible NSD, because she responded to steroids, and the symptoms disappeared except for slight hemiparesis and also met
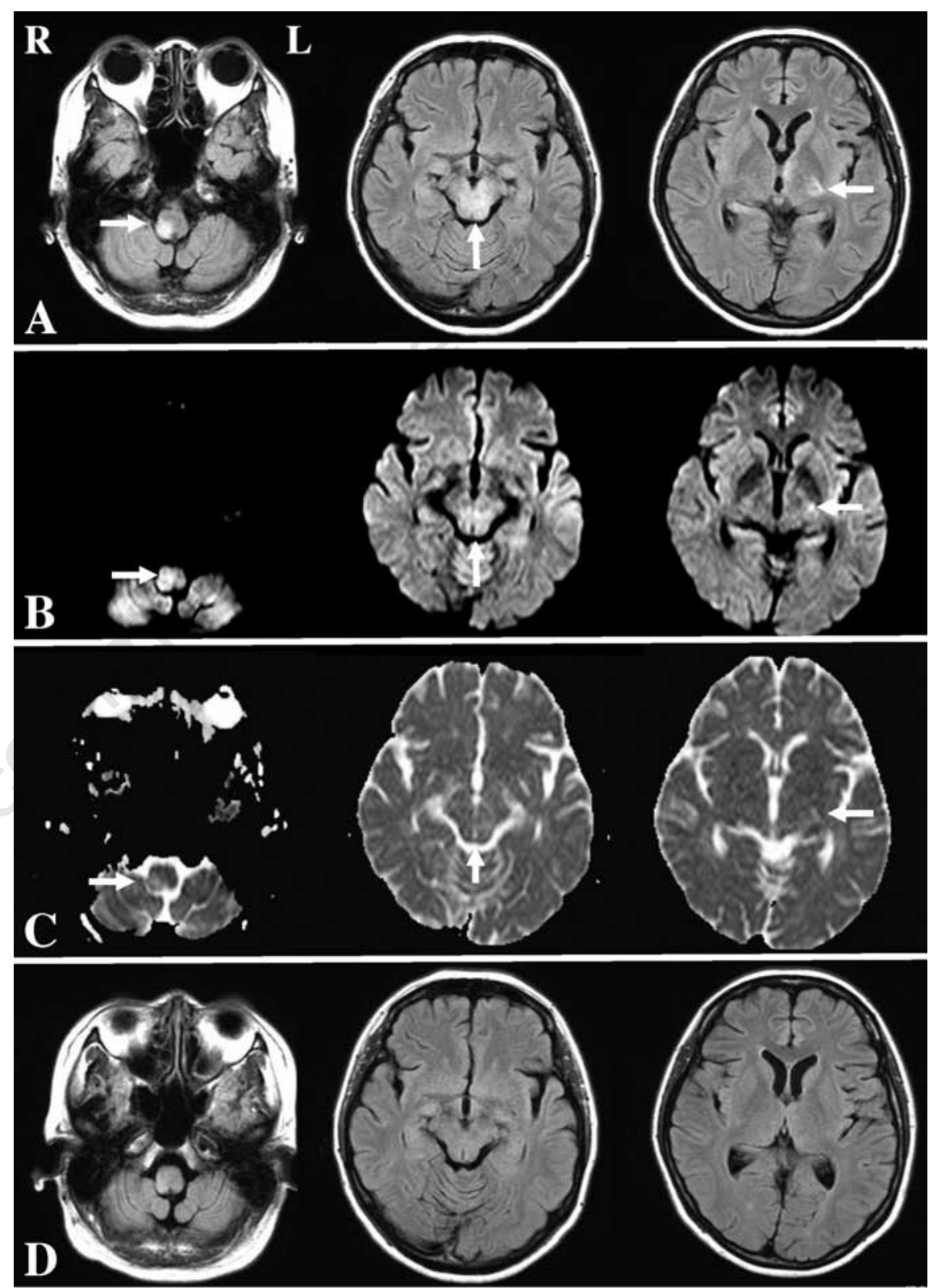

Figure 1. Brain MRI on admission in Case \#1. Serial images of axial fluid-attenuated inversion recovery magnetic resonance imaging and diffusion-weighted images showing high-intensity signals in the right dorsal medulla oblongata, bilateral dorsal midbrain, and left thalamus (A and B, arrows). Apparent diffusion coefficient values were increased (C, arrows). Follow-up FLAIR images at 20 days after admission showed that the highintensity signals in the right dorsal medulla oblongata, bilateral dorsal midbrain, and left thalamus were markedly decreased (D). 
other diagnostic criteria for NSD. NSD often initially presents with skin symptoms: Hisanaga et al. ${ }^{4}$ reported that 27 of 29 NSD patients initially presented with cutaneous symptoms or both cutaneous and neurological symptoms, and that only 2 initially presented with neurological symptoms Our search of the literature found that 39 patients with NSD had developed meningitis, ${ }^{5-12}$ but only 2 of them had initially presented with neurological symptoms, suggesting that such a case is rare (Table 1). In cases reported by Hisanaga et al. ${ }^{4}$ and Kokubo et al.,7 skin rashes appeared 29 and 64 months, respectively, after the onset of NSD; the diagnosis of NSD was established based on pathological findings. Their reports suggest that skin rashes may develop in our 2 surviving patients during their future clinical course.

Since there are currently no specific markers for NSD, it is difficult to differentiate NSD from infectious meningoencephalitis, multiple sclerosis, neuro- Behçet disease (NBD), Hashimoto's encephalopathy, Sjögren's syndrome, CNS lupus, sarcoidosis, non-herpetic limbic encephalitis, postviral encephalopathy, brain tumor, etc., before the results of HLA typing are known. In particular, the differentiation of NSD from NBD often poses a difficult diagnostic problem. These two diseases are considered to be closely related in that they are exudative immune diseases associated with a state of immune sensitivity, such as neutrophil hyperfunction. ${ }^{4}$ NSD is characterized by: i) little gender preponderance unlike the marked male preponderance of NBD; ii) age at onset between 30 and 70 years; iii) frequent recurrence but showing a favorable response to steroids and being less likely to result in sequelae; and iv) being almost always positive for HLA-Cw1 and frequently positive for HLAB54, unlike the high positive rate (about $80 \%$ ) of HLA-B51 in NBD. 13 In addition, NBD has a predilection for the basal ganglia and brainstem, whereas NSD presents with abnormal signal intensity areas at various sites of the central nervous system at similar frequencies, and these signal abnormalities often resolve with the relief of symptoms. Furthermore, it is thought that the lesions of NBD are often distributed ventrally in the brainstem corresponding to the clinical symptoms, whereas those of NSD are frequently located in the dorsal portion of the brainstem (mainly the mesencephalic tegmentum). The lesions of both diseases present a low intensity on T1-weighted images and high intensity on T2/FLAIR images, but the lesions of NBD and NSD often present with an isointensity and a high intensity, respectively. Moreover, acute active lesions of NBD are often enhanced with gadolinium, but NSD lesions are not. ${ }^{4}$ Both Patients \#1 and Patient \#2 were middle-aged women, and they responded well to steroid therapy with virtually no sequelae, suggesting that they had NSD. Also, the presence of highintensity areas in the dorsal portion of the
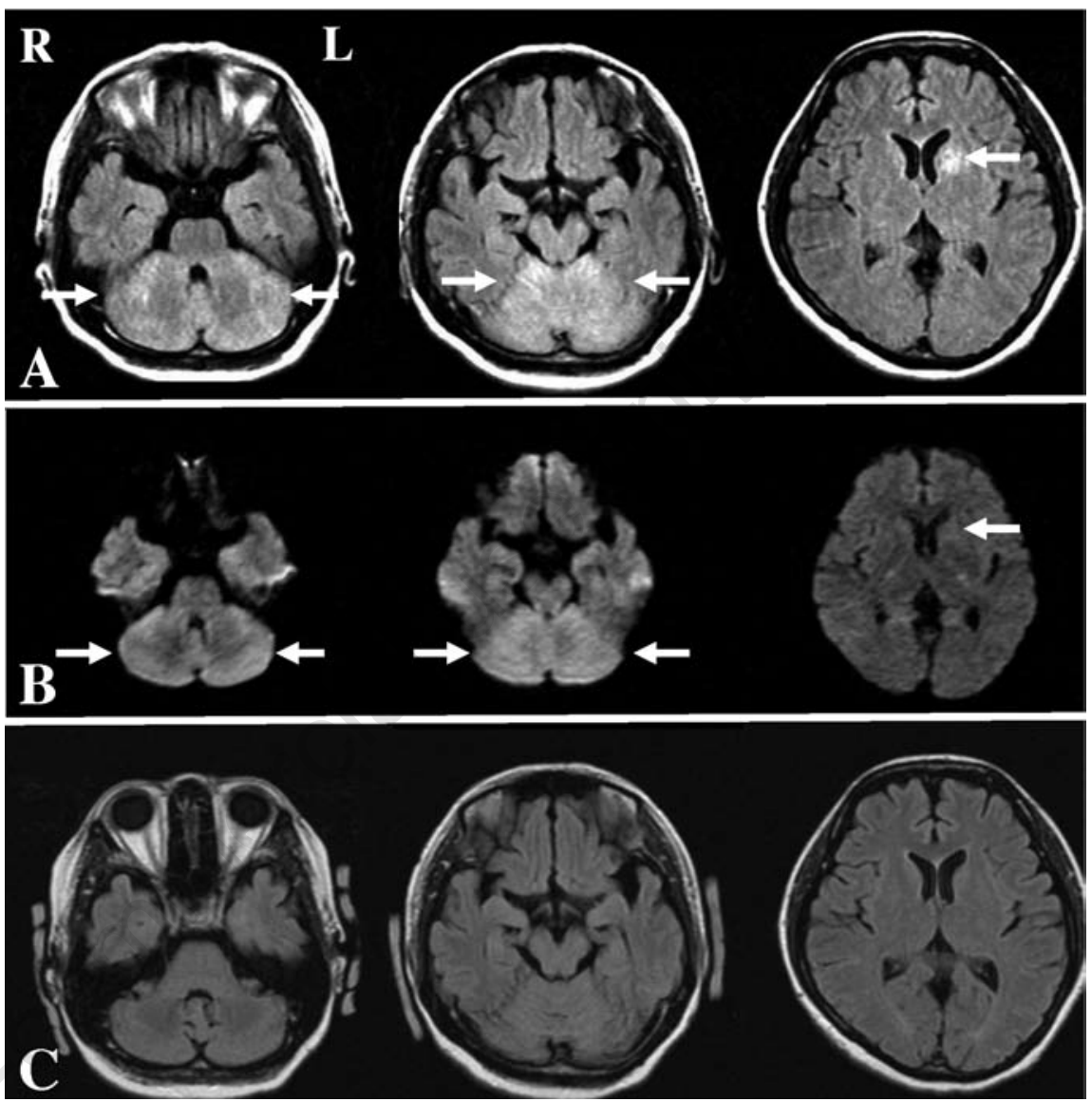

Figure 2. Brain magnetic resonance imaging 13 days after admission in Case \#2. Serial images of axial fluid-attenuated inversion recovery and diffusion-weighted images showing high-intensity signals in the bilateral cerebellar cortex and left caudate nucleus (A and $\mathrm{B}$, arrows). Follow-up fluid-attenuated inversion recovery images at 27 days after admission showed that the high-intensity signals in the bilateral cerebellar cortex and left caudate nucleus were markedly decreased (C).

Table 1. Clinical features of acute meningoencephalitis as the initial manifestation in neuro-Sweet disease.

\begin{tabular}{|c|c|c|c|c|c|c|c|}
\hline Case & Age/sex & Symptoms & MRI findings & $\begin{array}{c}\text { Time of dermal } \\
\text { events after } \\
\text { onset }\end{array}$ & Treatment & Outcome & $\begin{array}{l}\text { Author } \\
\text { report } \\
\text { year, ref. }\end{array}$ \\
\hline 1 & $32 / \mathrm{M}$ & $\begin{array}{l}\text { Disorientation, } \\
\text { fever }\end{array}$ & $\begin{array}{l}\text { Basal ganglia, brain stem, } \\
\text { cerebral white matter }\end{array}$ & 29 months & Steroid & Favorable & $\begin{array}{c}\text { Hisanaga et al. } \\
19994\end{array}$ \\
\hline 2 & $34 / \mathrm{M}$ & Dysarthria, hemiparesis & $\begin{array}{l}\text { Basal ganglia, brain stem, } \\
\text { cerebral white matter }\end{array}$ & 64 months & Steroid & Favorable & Kokubo et al. $2006^{7}$ \\
\hline 3 & $51 / \mathrm{F}$ & $\begin{array}{l}\text { Dizziness, headache, } \\
\text { fever }\end{array}$ & Brain stem, thalamus & & Steroid & Favorable & $\begin{array}{l}\text { Our case \#1 } \\
\quad 2011\end{array}$ \\
\hline 4 & $42 / \mathrm{F}$ & Headache, fever & Basal ganglia, cerebellum & & Steroid & Favorable & $\begin{array}{l}\text { Our case \#2 } \\
2011\end{array}$ \\
\hline
\end{tabular}


medulla oblongata of Patient \#1 on FLAIR and diffusion-weighted images was suggestive of NSD. In addition, an increase in the apparent diffusion coefficient was observed in the lesions, suggesting reversible extracellular edema. Conversely, an HLA-B54-positive patient was conclusively diagnosed with NBD by skin biopsy. 14 Therefore, not only HLA typing but also clinical symptoms and histopathological examination are important for the differentiation between NBD and NSD, and patients should be carefully followed-up, especially for the development of skin rashes.

\section{References}

1. Cohen PR, Kurzrock R. Sweet's syndrome revisited: a review of disease concepts. Int J of Dermatol 2003;42:761-78.

2. Cohen PR. Sweet's syndrome - a comprehensive review of an acute febrile neutrophilic dermatosis. Orphanet J Rare Dis 2007;2:34.

3. Hisanaga K, Iwasaki Y, Itoyama Y. The Neuro-Sweet Disease Study Group. Neuro-
Sweet disease: clinical manifestations and criteria for diagnosis. Neurology 2005;64: 1756-61.

4. Hisanaga K, Hosokawa M, Sato N, et al. "Neuro-Sweet disease": benign recurrent encephalitis with neutrophilic dermatosis. Arch Neurol 1999;56:1010-3.

5. Noda K, Okuma Y, Fukae J, et al. Sweet's syndrome associated with encephalitis. J Neurol Sci 2001;188:95-7.

6. Tsunemi T, Sakai Y, Tsunoda K, et al. Neuro-Behçet's/neuro-Sweet's disease presents simultaneously with severe tonsillitis, and features mimicking bacterial meningitis with skin lesions. Intern Med 2006;45:1315-7.

7. Kokubo Y, Kuzuhara S, Isoda $\mathrm{K}$, et al. Neuro-Sweet disease: report of the first autopsy case. J Neurol Neurosurg Psychiatry 2007;78:997-1000.

8. Song IU, Kim JS, Kim YI, Lee KS. Sweet's syndrome associated with acute-onset encephalitis. Eur Neurol 2007;57:182-4.

9. Fukae J, Noda K, Fujishima K, et al. Successful treatment of relapsing neuroSweet's disease with corticosteroid and dapsone combination therapy. Clin Neurol Neurosurg 2007;109:910-3.
10. Kimura A, Sakurai T, Koumura A, et al. Longitudinal analysis of cytokines and chemokines in the cerebrospinal fluid of a patient with neuro-Sweet disease presenting with recurrent encephalomeningitis. Intern Med 2008;47:135-41.

11. Aydemir H, Öztoprak N, Celebi G, et al. Pulmonary and central nervous system involvement in Sweet's syndrome: a very rare case report. Intern Med 2008;47:14814.

12. Fukushima K, Hineno A, Kodaira M, et al. Reversible extensive leukoencephalopathy in Sweet disease: a case report. J Neurol Sci 2008;275:178-80.

13. Mizoguchi M, Matsuki K, Mochizuki M, et al. Human leukocyte antigen in Sweet's syndrome and its relationship to Behçet's disease. Arch Dermatol 1988;124:1069-73.

14. Sato M, Yoneda M, Kumakiri M, et al. A case of neruo-Behçet disease with HLAB54, Cw1, which is difficult clinically in the differential diagnosis with neuroSweet disease. Clin Neurol 2009;49:47982. 